\title{
Prior antithrombotic use is significantly associated with decreased blood viscosity within 24 hours of symptom onset in patients with acute ischemic stroke
}

\author{
Journal of \\ Neurocritical \\ Care \\ ORIGINAL ARTICLE \\ Received: July 30, 2019 \\ Revised: September 3, 2019 \\ Accepted: September 3, 2019 \\ Corresponding Author: \\ Sang Won Han, MD \\ Department of Neurology, Inje \\ University Sanggye Paik Hospital, Inje \\ University College of Medicine, 1342 \\ Dongil-ro, Nowon-gu, Seoul 01757, \\ Republic of Korea \\ Tel: +82-2-950-4832 \\ Fax: +82-2-950-1955 \\ E-mail: swhan@paik.ac.kr
}

Joong Hyun Park, MD; Jeong Yeon Kim, MD, PhD'; Jong Sam Baik, MD, PhD; Jae Hyeon Park, MD, PhD'; Hyo Suk Nam, MD, PhD²; Sang Won Han, MD

'Department of Neurology, Inje University Sanggye Paik Hospital, Inje University College of Medicine, Seoul, Republic of Korea

${ }^{2}$ Department of Neurology, Yonsei University College of Medicine, Seoul, Republic of Korea

Background: Blood viscosity (BV) is the intrinsic resistance of blood to flow and is a measure of blood stickiness. Several clinical and epidemiologic studies have demonstrated an association between BV and the occurrence of major thromboembolic events. Although $\mathrm{BV}$ is significantly higher in cases of lacunar or cardioembolic strokes, its relationship with demographic and laboratory parameters during the acute stage of ischemic stroke is unknown. We investigated the relationship between baseline characteristics of acute ischemic stroke and BV within 24 hours of symptom onset in patients with acute ischemic stroke.

Methods: We enrolled patients aged 40 years or older with documented histories of ischemic stroke or transient ischemic attack within 24 hours of symptom onset. A scanning capillary-tube viscometer was used to assess whole BV.

Results: The mean age was $69.6 \pm 12.03$ years and $44.4 \%$ of the patients were female. Of 189 patients, $68.3 \%$ had a history of hypertension; 27\%, diabetes; $42.9 \%$, hypercholesterolemia; $3.7 \%$, coronary artery disease; and $18 \%$, stroke. Additionally, $40.7 \%$ were current smokers. Sixty-one patients (32.3\%) were regularly taking antithrombotics. Multiple linear regression analysis revealed that hematocrit was positively corelated with increased BV and prior antithrombotic use was corelated with decreased BV. Hematocrit-adjusted partial correlation demonstrated that prior antithrombotic use was significantly associated with decreased BV.

Conclusion: Prior antithrombotic use is significantly associated with decreased BV within 24 hours of symptom onset in patients with acute ischemic stroke. Our findings indicate that antithrombotic medications may change the hemorheological profile in these patients.

Keywords: Aspirin; Blood viscosity; Hematocrit; Blood platelets; Stroke

\section{INTRODUCTION}

Blood viscosity (BV) is defined as the intrinsic resistance of blood to flow and serves as a measure of blood "stickiness" [1-3]. BV is an essential hemorheological factor and is determined by hematocrit, plasma viscosity (PV), and properties of red blood cells (RBCs) [4]. Increases in these factors correspond to increases in BV. High aggregation and low deformability of RBCs can also increase BV. High BV, in turn, increases thromboembolic risk and plays an important role in cerebro- cardiovascular diseases $[2,3,5-7]$. Several

(C) 2019 The Korean Neurocritical Care Society

This is an Open Access article distributed under the terms of the Creative Commons Attribution Non-Commercial License (http://creativecommons.org/licenses/by-nc/4.0/) which permits unrestricted noncommercial use, distribution, and reproduction in any medium, provided the original work is properly cited. 
clinical and epidemiologic studies have demonstrated an association between $\mathrm{BV}$ and the occurrence of major thromboembolic events $[8,9]$. BV can also be altered during various physiopathological conditions including obesity, cigarette smoking, chronic heart failure, hypertension, and diabetes [10]. Furthermore, BV can be modified using medical treatments such as antithrombotics or statins. Although high BV is known to contribute to stroke occurrence, few studies have examined BV in acute ischemic stroke $[1,3,10,11]$. There is evidence to show that BV is significantly higher in cases of lacunar or cardioembolic strokes $[1,3,12]$. However, the relationship between BV and biochemical parameters during the acute stage of ischemic stroke have not yet been elucidated. Therefore, we investigated the relationship between baseline characteristics of acute ischemic stroke and BV within 24 hours of symptom onset in such patients.

\section{METHODS}

\section{Patients}

We enrolled patients aged 40 years or older with documented histories of ischemic stroke or transient ischemic attack (TIA) within 24 hours of symptom onset between January 2018 and December 2018. In order to be eligible for inclusion in the study, the symptom complex of TIA patients had to encompass weakness, speech disturbance, dysarthria or dysphasia for greater than 5 minutes [13]. Patient demographics, clinical information including vascular risk factors, and medical histories were assessed during hospital admission. A skilled pharmacist checked the medications each patient took regularly during the week preceding their admission. The laboratory findings affecting BV, including hemoglobin, hematocrit, white blood cells, platelets, random plasma glucose, and prothrombin time-international normalized ratio (PT-INR) were examined during initial blood sampling in the emergency room (ER). Blood protein, fasting blood sugar, and lipid profiles were obtained after a 12-hour fast. All patients underwent systemic investigations of brain magnetic resonance imaging and at least one vascular imaging study, such as conventional angiography, magnetic resonance angiography, or computed tomographic angiography. Echocardiography and 24-hour Holter monitoring were done in a patient with embolic stroke of undetermined etiology to detect the cardioembolic source. Stroke subtypes were assigned according to the Trial of ORG 10172 in the Acute Stroke Treatment classification system, and the criteria for classification were strictly enforced. We excluded patients diagnosed with stroke subtypes of other determined etiologies, such as nonatherosclerotic vasculopathy, hypercoagulable states, and hematologic disorders.

\section{BV measurement}

A scanning capillary-tube viscometer (SCTV) (Hemovister, Pharmode Inc., Seoul, Korea) was used to assess the whole blood viscosity (WBV). The SCTV assesses systolic WBV (SBV) and diastolic WBV (DBV). SBV and DBV characterize viscosities at high and low shear rates, respectively. A WBV measured at a shear rate of 300 second $^{-1}$ was selected as the SBV and at 1 second $^{-1}$ as the DBV [3]. A $3 \mathrm{~mL}$ of whole blood from each patient was collected in an ethylenediaminetetraacetic acid anticoagulant-coated tube and stored at $4^{\circ} \mathrm{C}$. All BV levels were obtained before hydration therapy in the ER, and measurements were taken within 24 hours of collection.

\section{Statistical analysis}

Variables were tested for normality using the Kolmogorov-Smirnov test. The baseline parameters of each group were analyzed using one-way analysis of variance (ANOVA) with a Tukey post hoc test for continuous variables wherever applicable. Univariate analyses of demographics, vascular risk factors, and medical history were performed using an independent sample $t$ test or the Mann-Whitney $U$ test for continuous variables and the chi-square test for categorical variables. Multivariate linear regression models were used to investigate the relationship between significant univariate variables. A multivariate analysis was performed using all variables with $P<0.05$ in the univariate analysis. Descriptive data were expressed as number (percent) or mean \pm standard deviation. Statistical analyses were performed using SPSS version 25.0 for Windows (IBM Co., Armonk, NY, USA).

\section{Institutional Review Board/Institutional Animal Care and Use Committee approval}

The Research Ethics Committee of Inje University Sanggye Paik Hospital approved the present study (2018-08-025). The requirement for informed consent was waived because the database was accessed only for purposes of analysis; personal information was not used.

\section{RESULTS}

The patient profile and reasons for exclusion from the study have been outlined in Fig. 1. Of 189 patients, 22 had TIA (11.6\%). The most frequent stroke subtype was lacunar stroke $(n=65,34.4 \%)$, followed by stroke of undetermined etiology, negative workup $(n=43,22.8 \%)$, large artery atherosclerosis $(n=32,16.9 \%)$, and cardioembolism (CE) $(n=27,14.3 \%)$. The baseline characteristics of the study population are shown in Table 1 . The mean age was $69.6 \pm 12.03$ years, and $44.4 \%$ were female. Of these, $68.3 \%$ 


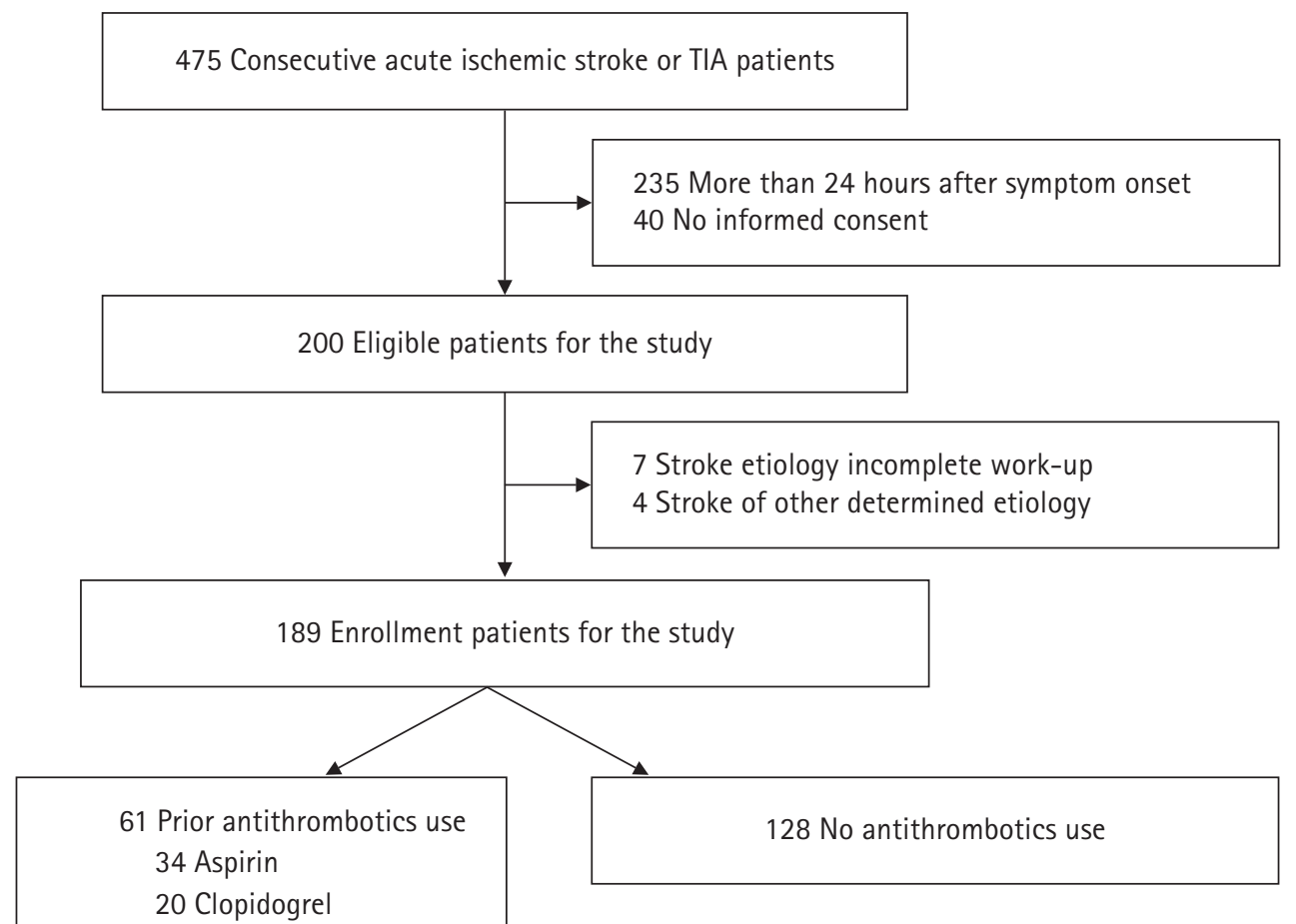

Fig. 1. Trial profile. TIA, transient ischemic attack.

had a history of hypertension; $27 \%$, diabetes; $42.9 \%$, hypercholesterolemia; 3.7\%, coronary artery disease; and 18\%, stroke. Additionally, $40.7 \%$ were smokers at the time of the study. Sixty-one patients $(32.3 \%)$ were regularly taking antithrombotics (aspirin, 56\%; clopidogrel, 33\%; warfarin, 11\%). There were no patients taking dual antiplatelet therapy or a new oral anticoagulant. Older age was highly correlated with antithrombotic use $(r=0.324$, $P<0.0001)$. Though INR was higher in patients with prior antithrombotic use (1.09 vs. $1.01, P=0.045$ ), there was no difference in INR between antiplatelet and anticoagulant user groups (1.09 vs. 1.14, $P=0.645$ ). Patients with CE were older, had higher INR, lower smoking rates, and lower low-density lipoprotein cholesterol (LDL-C) levels, likely related to the higher number of female patients and higher use of antithrombotics among patients in this category (Table 1). Table 2 shows the difference in BV based on the history of prior antithrombotic use. Prior antithrombotic use was positively associated with decreased BV but no significant differences in BV were observed among treatment groups.

Univariate linear regression analyses were performed on baseline characteristics relative to SBV and DBV. SBV and DBV were significantly associated with age, smoking, hemoglobin, hematocrit, white blood cell count, platelet count, total cholesterol level, LDL-C level, and prior antithrombotic use. Prior statin use did not influence BV levels (SBV, $P=0.485$ vs. DBV, $P=0.766$ ). Multiple linear regression analysis revealed that hematocrit, platelet count, and prior antithrombotic use were associated with SBV and DBV (Table 3). Hematocrit was positively related with increased SBV, and hematocrit and platelet count were positively associated with increased DBV. Prior antithrombotic use was significantly related with decreased SBV and DBV. Hematocrit-adjusted partial correlation revealed that prior antithrombotic use was significantly associated with decreased SBV and DBV ( $r=-$ $0.227, P=0.014$ vs. $r=-0.231, P=0.013$ ) (Fig. 2).

\section{DISCUSSION}

$\mathrm{BV}$ reflects frictional interactions between $\mathrm{RBCs}$ and other blood components within the systemic vascular system [2]. The major determinants of $\mathrm{BV}$ are the aggregation and deformability of RBCs, hematocrit, and PV. BV can provide important information regarding stroke risk and can be modified by therapeutic modalities. In this study, we investigated the relationship between patient characteristics and BV within 24 hours of symptom onset in acute ischemic stroke. We found that prior antithrombotic use was associated with decreased BV.

As discussed earlier, aggregation and deformability of RBCs are some of the main factors that influence BV. Upon reaching the arterioles, $\mathrm{RBC}$ aggregates are dispersed due to increased shear. Once they pass through the arterioles, RBCs flow as individual cells through the capillaries. After capillary passage, they again ag- 
Table 1. Baseline characteristics of the study population

\begin{tabular}{|c|c|c|c|c|c|c|c|}
\hline Characteristic & Total $(n=189)$ & LAA $(n=32)$ & Lacune $(n=65)$ & SUDn $(n=43)$ & CE $(n=27)$ & TIA $(n=22)$ & $P$ value \\
\hline \multicolumn{8}{|l|}{ Demographic } \\
\hline Age (yr) & $69.6 \pm 12.03$ & $71.9 \pm 11.28$ & $66.7 \pm 10.87$ & $71.1 \pm 12.32$ & $76.9 \pm 9.51$ & $62.8 \pm 13.32$ & $<0.001^{a)}$ \\
\hline Female sex & $84(44.4)$ & $11(34.4)$ & $27(43.5)$ & $20(35.8)$ & $14(51.9)$ & $12(54.5)$ & 0.538 \\
\hline \multicolumn{8}{|l|}{ Medical history } \\
\hline Hypertension & $129(68.3)$ & $20(62.5)$ & $44(67.7)$ & $30(69.8)$ & 24 (88.9) & $11(50)$ & 0.056 \\
\hline Diabetes mellitus & $51(27)$ & $11(34.4)$ & $21(32.3)$ & 8 (18.6) & 9 (33.3) & $2(9.1)$ & 0.113 \\
\hline Hypercholesterolemia & $81(42.9)$ & $13(40.6)$ & $29(44.6)$ & 15 (34.9) & $15(55.6)$ & $9(40.9)$ & 0.545 \\
\hline Stroke & 34 (18) & 7 (21.9) & $11(16.9)$ & $6(14)$ & $9(33.3)$ & $1(4.5)$ & 0.097 \\
\hline Coronary artery disease & 7 (3.7) & $1(3.1)$ & $1(1.5)$ & $0(0)$ & $4(14.8)$ & $1(4.5)$ & 0.087 \\
\hline Smoking & $77(40.7)$ & $18(56.2)$ & $32(49.2)$ & $15(34.9)$ & $5(18.5)$ & 7 (31.8) & 0.017 \\
\hline Antithrombotic use & $61(32.3)$ & $10(31.2)$ & $17(26.2)$ & 9 (20.9) & $20(74.1)$ & $5(22.7)$ & $<0.001^{\mathrm{a})}$ \\
\hline Stain use & $62(32.8)$ & $9(28.1)$ & $20(30.8)$ & $15(55.6)$ & $11(26.2)$ & 7 (31.8) & 0.150 \\
\hline \multicolumn{8}{|l|}{ Laboratory finding } \\
\hline Hemoglobin (g/dL) & $13.7 \pm 2.05$ & $13.7 \pm 2.45$ & $13.9 \pm 1.69$ & $13.7 \pm 2.05$ & $13.2 \pm 2.24$ & $14 \pm 1.85$ & 0.745 \\
\hline Hematocrit (\%) & $40.8 \pm 5.73$ & $40.9 \pm 6.27$ & $40.9 \pm 4.6$ & $41 \pm 5.73$ & $39.5 \pm 6.29$ & $41.4 \pm 5.12$ & 0.828 \\
\hline White blood cells $\left(10^{3} / \mu \mathrm{L}\right)$ & $8.01 \pm 3.09$ & $9.21 \pm 4.02$ & $7.07 \pm 2.23$ & $8.14 \pm 3.11$ & $9.08 \pm 3.04$ & $7.34 \pm 2.95$ & 0.316 \\
\hline Platelets $\left(10^{3} / \mu \mathrm{L}\right)$ & $238 \pm 70.5$ & $269 \pm 86.23$ & $234 \pm 66.43$ & $234 \pm 58.51$ & $223 \pm 75.77$ & $228 \pm 61.39$ & 0.300 \\
\hline Random plasma glucose (mg/dL) & $145 \pm 60.04$ & $137 \pm 48.64$ & $153 \pm 65.85$ & $136 \pm 62.7$ & $160 \pm 62.22$ & $129 \pm 42.98$ & 0.262 \\
\hline Total cholesterol (mg/dL) & $157 \pm 38.24$ & $152 \pm 36.73$ & $168 \pm 40.49$ & $152 \pm 35.79$ & $146 \pm 36.52$ & $154 \pm 36.05$ & 0.087 \\
\hline $\mathrm{LDL}-\mathrm{C}(\mathrm{mg} / \mathrm{dL})$ & $97 \pm 29.06$ & $96 \pm 26.85$ & $106 \pm 30.71$ & $94 \pm 27.93$ & $85 \pm 25.14$ & $95 \pm 28.74$ & $0.027^{a)}$ \\
\hline $\mathrm{HDL}-\mathrm{C}(\mathrm{mg} / \mathrm{dL})$ & $40 \pm 7.25$ & $39 \pm 9.26$ & $41 \pm 8.23$ & $40 \pm 9.47$ & $44 \pm 13.38$ & $41 \pm 7.25$ & 0.492 \\
\hline Triglyceride (mg/dL) & $121 \pm 113.59$ & $106 \pm 39.01$ & $129 \pm 68.77$ & $119 \pm 59.45$ & $116 \pm 114.8$ & $132 \pm 62.59$ & 0.684 \\
\hline INR & $1.04 \pm 0.18$ & $1.03 \pm 0.65$ & $0.99 \pm 0.64$ & $1.02 \pm 0.79$ & $1.18 \pm 0.4$ & $1.04 \pm 0.64$ & $<0.009^{a)}$ \\
\hline $\mathrm{SBV}(\mathrm{cP})$ & $4.39 \pm 0.91$ & $4.47 \pm 1.02$ & $4.49 \pm 0.77$ & $4.23 \pm 0.87$ & $4.42 \pm 1.11$ & $4.26 \pm 0.94$ & 0.574 \\
\hline $\mathrm{DBV}(\mathrm{cP})$ & $13.66 \pm 3.15$ & $13.91 \pm 3.42$ & $14.06 \pm 2.74$ & $13.08 \pm 3.01$ & $13.76 \pm 3.83$ & $13.12 \pm 3.27$ & 0.498 \\
\hline
\end{tabular}

Values are presented as mean \pm SD or number $(\%)$.

LAA, large artery atherosclerosis; SUDn, stroke of undetermined etiology, negative work-up; CE, cardioembolism; TIA, transient ischemic attack; LDL-C, low-density lipoprotein cholesterol; HDL-C, high-density lipoprotein cholesterol; INR, international normalized ratio; SBV, systolic whole blood viscosity; $\mathrm{CP}$, centipoise; $\mathrm{DBV}$, diastolic whole blood viscosity.

${ }^{a}$ Significant $P$ values.

Table 2. Differences in blood viscosity based on prior use of antithrombotics

\begin{tabular}{lrrrr}
\hline Variable & SBV (cP) & $P$ value & DBV (cP) & $P$ value \\
\hline No antithrombotics $(n=128)$ & $4.5 \pm 0.96$ & & $14.1 \pm 3.39$ & \\
Prior antithrombotics $(n=61)$ & $4.1 \pm 0.56$ & $\left.0.001^{\mathrm{a}}\right)$ & $12.6 \pm 2.13$ & $0.003^{\mathrm{a})}$ \\
$\quad$ Antiplatelets $(\mathrm{n}=54)$ & $4 \pm 0.58$ & $0.002^{\mathrm{a}}$ & $12.5 \pm 2.17$ & $\left.0.003^{\mathrm{a}}\right)$ \\
$\quad$ Aspirin $(\mathrm{n}=34)$ & $4 \pm 0.54$ & $0.001^{\mathrm{a})}$ & $12.5 \pm 2.37$ & $0.002^{\mathrm{a})}$ \\
$\quad$ Clopidogrel $(\mathrm{n}=20)$ & $4.1 \pm 0.59$ & $0.002^{\mathrm{a}}$ & $12.5 \pm 2.04$ & $0.003^{\mathrm{a})}$ \\
$\quad$ Warfarin $(\mathrm{n}=7)$ & $4.1 \pm 0.47$ & 0.363 & $13 \pm 1.98$ & 0.447 \\
Antiplatelets vs. Warfarin & & 0.709 & & 0.603 \\
Aspirin vs. Warfarin & & 0.725 & & 0.804 \\
Clopidogrel vs. Warfarin & & 0.734 & & 0.826 \\
Aspirin vs. Clopidogrel & & 0.897 & & 0.872 \\
\hline
\end{tabular}

Values are presented as mean \pm SD.

SBV, systolic whole blood viscosity; CP, centipoise; DBV, diastolic whole blood viscosity.

${ }^{\text {a) }}$ Significant $P$ values.

gregate within collecting venules [2]. Factors that increase RBC aggregation also increase flow resistance in microcirculation. In this scenario hematocrit, the volume fraction of RBCs in whole blood, becomes a vital factor that affects BV. Changes in the he- 
Table 3. Multivariate linear regression analyses of baseline characteristics with regard to SBV and DBV

\begin{tabular}{|c|c|c|c|c|c|c|}
\hline \multirow{2}{*}{ Variable } & \multicolumn{3}{|c|}{ SBV } & \multicolumn{3}{|c|}{ DBV } \\
\hline & $\mathrm{B}(\mathrm{SE})$ & Standardized $\beta$ & $P$ value & $\mathrm{B}(\mathrm{SE})$ & Standardized $\beta$ & $P$ value \\
\hline Age & $0.007(0.005)$ & 0.096 & 0.156 & $0.021(0.016)$ & 0.085 & 0.190 \\
\hline Smoking & $-0.01(0.109)$ & -0.006 & 0.924 & $-0.174(0.368)$ & -0.028 & 0.638 \\
\hline Hemoglobin & $0.005(0.037)$ & 0.029 & 0.900 & $0.037(0.124)$ & 0.066 & 0.768 \\
\hline Hematocrit & $0.329(0.104)$ & 0.758 & $0.002^{\text {a) }}$ & $1.147(0.35)$ & 0.749 & $0.001^{\mathrm{a})}$ \\
\hline White blood cells & $0.029(0.016)$ & 0.106 & 0.072 & $0.089(0.054)$ & 0.093 & 0.099 \\
\hline Platelets & $0.001(0.001)$ & 0.113 & 0.073 & $0.005(0.002)$ & 0.135 & $0.026^{a)}$ \\
\hline Total cholesterol & $-0.001(0.005)$ & -0.042 & 0.843 & $-0.001(0.016)$ & -0.011 & 0.955 \\
\hline LDL-C & $0.003(0.006)$ & 0.101 & 0.633 & $0.005(0.022)$ & 0.048 & 0.812 \\
\hline Antithrombotic use & $-0.259(0.109)$ & -0.135 & $0.019^{a)}$ & $-0.892(0.368)$ & -0.132 & $0.017^{\mathrm{a})}$ \\
\hline
\end{tabular}

SBV, systolic whole blood viscosity; DBV, diastolic whole blood viscosity; SE, standard error; LDL-C, low-density lipoprotein cholesterol.

Significant $P$ values.
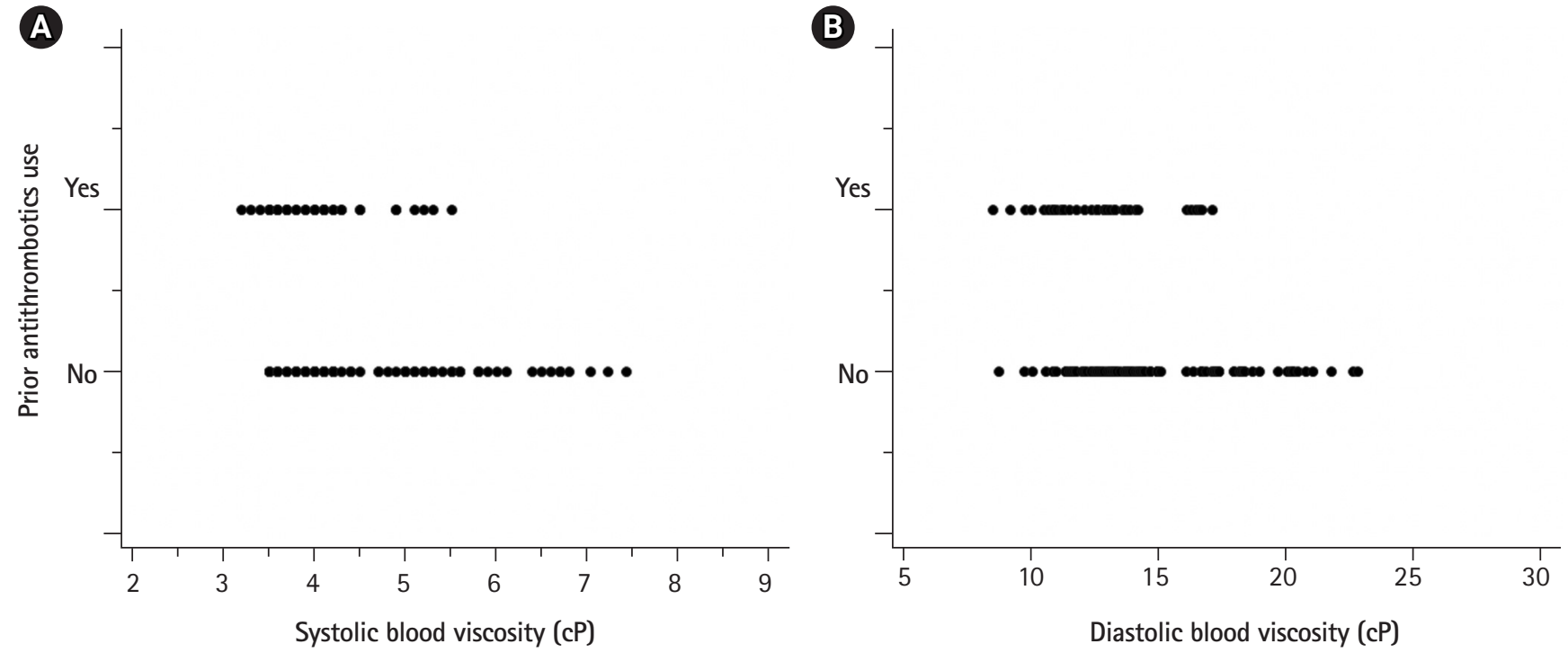

Fig. 2. (A, B) Scatterplots of the relationship between prior antithrombotic use and blood viscosity (BV). Hematocrit-adjusted partial correlation shows that prior antithrombotic use is significantly associated with decreased systolic and diastolic whole BV ( $r=-0.227$, $P=0.014$ vs. $r=-0.231, P=0.013)$. $c P$, centipoise; $r$, Pearson's partial correlation coefficient.

matocrit significantly contributes to rheological discrepancies. A $10 \%$ increase in hematocrit increases the SBV by $5 \%$, whereas the same increase in hematocrit increases the DBV by $30 \%$ [14]. Due to the variations of hematocrit in small vessels, its influence on BV is more pronounced in the microcirculation [15]. One of the more conspicuous impacts of $\mathrm{BV}$ is on microcirculatory tissue perfusion. A hematocrit-induced increase in BV may lead to a decrease in tissue perfusion.

BV can be modified using drugs such as vasodilators, statins, or antithrombotics $[2,16,17]$. A few studies have examined the effect of antithrombotics on BV [17-19] and found that warfarin, heparin, and argatroban can decrease BV $[17,20]$. Varying results have been observed with antiplatelets, depending on the experimental protocol used in the study. Aspirin and cilostazol do not change $\mathrm{BV}$ but dipyridamole and clodogrel decrease BV upon treatment $[18,21,22]$. Antithrombotics may decrease BV by inhibiting platelet aggregation and improving RBC deformability. Our results showed that prior antithrombotic use was significantly associated with decreased BV. The use of both anticoagulants and antiplatelets were related to a decrease in BV. Although we could not find plausible explanations for these discrepancies among studies, patient-specific differences may be a contributing factor. We studied patients with an ischemic stroke or TIA within 24 hours of symptom onset. Informative studies of $\mathrm{BV}$ and acute ischemic stroke 
are limited and previous studies have not examined this period $[1,3,10]$. Further studies focusing on this specific time period in ischemic stroke patients are required to assess the influence of prior antithrombotic use and BV. An older study has demonstrated significantly lower cerebral blood flow (CBF) in patients with hematocrit in the range of $47 \%$ to $53 \%$ than in a group with $36 \%$ to $46 \%$ [23]. In the former group, phlebotomy increased CBF, implying that optimal hematocrit and BV levels may exist for CBF. Another factor that is possibly associated with increased $\mathrm{BV}$ is dehydration. One study showed that BV at admission was significantly higher in patients with lacunar stroke but normalized after 2 weeks of normal hydration [1]. In our study, all blood samples for BV measurement were collected before hydration therapy.

The present study had several limitations. This was a small cross-sectional and observational study, implying the existence of concealed confounders. We could not measure plasma components such as fibrinogen or $\mathrm{C}$-reactive protein in all patients, both of which can affect BV. We enrolled only Korean patients, which limited our ability to generalize the inferences of the study to non-Korean patients. In concordance with our multivariate analysis results, previous studies show that antithrombotics reduce the $\mathrm{BV}$ significantly in patients with acute ischemic stroke. However, the statistical power to support our conclusion is fairly weak, mainly due to the small sample size and lack of age-matched controls. These limitations should be considered when interpreting our data.

In conclusion, we found that prior antithrombotic use is significantly associated with decreased BV within 24 hours of symptom onset in patients with acute ischemic stroke. Our results indicate that antithrombotic medications may change the hemorheological profile in acute ischemic stroke patients. Further studies are essential to evaluate the role of $\mathrm{BV}$ plus antithrombotic therapy on the risk of stroke occurrence.

\section{ARTICLE INFORMATION}

\section{Conflict of interest}

No potential conflict of interest relevant to this article.

\section{ORCID}

Joong Hyun Park, https://orcid.org/0000-0001-6118-4833 Jeong Yeon Kim, https://orcid.org/0000-0002-7106-2773 Jong Sam Baik, https://orcid.org/0000-0002-5300-203X Jae Hyeon Park, https://orcid.org/0000-0003-4523-9384 Hyo Suk Nam, https://orcid.org/0000-0002-4415-3995 Sang Won Han, https://orcid.org/0000-0002-9503-1883

\section{Author contributions}

Conceptualization: SWH. Data curation \& Formal analysis: JHP and SWH. Funding acquisition: JHP. Investigation: JHP and SWH. Methodology: JHP and SWH. Project administration: JHP. Resources: JHP. Software: JYK. Supervision: JSB. Validation: JSB. Visualization \& Writing-original draft: HSN and JHP. Writing-review editing: $\mathrm{HSN}$ and SWH.

\section{REFERENCES}

1. Furukawa K, Abumiya T, Sakai K, Hirano M, Osanai T, Shichinohe $\mathrm{H}$, et al. Increased blood viscosity in ischemic stroke patients with small artery occlusion measured by an electromagnetic spinning sphere viscometer. J Stroke Cerebrovasc Dis 2016;25:2762-9.

2. Pop GA, Duncker DJ, Gardien M, Vranckx P, Versluis S, Hasan $\mathrm{D}$, et al. The clinical significance of whole blood viscosity in (cardio)vascular medicine. Neth Heart J 2002;10:512-6.

3. Song SH, Kim JH, Lee JH, Yun YM, Choi DH, Kim HY. Elevated blood viscosity is associated with cerebral small vessel disease in patients with acute ischemic stroke. BMC Neurol 2017; 17:20.

4. Baskurt OK, Meiselman HJ. Blood rheology and hemodynamics. Semin Thromb Hemost 2003;29:435-50.

5. Kwaan HC. Role of plasma proteins in whole blood viscosity: a brief clinical review. Clin Hemorheol Microcirc 2010;44:16776.

6. Mury P, Faes C, Millon A, Mura M, Renoux C, Skinner S, et al. Higher daily physical activity level is associated with lower $\mathrm{RBC}$ aggregation in carotid artery disease patients at high risk of stroke. Front Physiol 2017;8:1043.

7. Noh HJ, Seo SW, Jeong Y, Park JE, Kim GH, Noh Y, et al. Blood viscosity in subcortical vascular mild cognitive impairment with versus without cerebral amyloid burden. J Stroke Cerebrovasc Dis 2014;23:958-66.

8. Cowan AQ, Cho DJ, Rosenson RS. Importance of blood rheology in the pathophysiology of atherothrombosis. Cardiovasc Drugs Ther 2012;26:339-48.

9. Danesh J, Collins R, Peto R, Lowe GD. Haematocrit, viscosity, erythrocyte sedimentation rate: meta-analyses of prospective studies of coronary heart disease. Eur Heart J 2000;21:515-20.

10. Grotemeyer KC, Kaiser R, Grotemeyer KH, Husstedt IW. Association of elevated plasma viscosity with small vessel occlusion in ischemic cerebral disease. Thromb Res 2014;133:96100.

11. Cecchi E, Marcucci R, Poli D, Antonucci E, Abbate R, Gensini GF, et al. Hyperviscosity as a possible risk factor for cerebral 
ischemic complications in atrial fibrillation patients. Am J Cardiol 2006;97:1745-8.

12. Fisher M, Meiselman HJ. Hemorheological factors in cerebral ischemia. Stroke 1991;22:1164-9.

13. Kennedy J, Hill MD, Ryckborst KJ, Eliasziw M, Demchuk AM, Buchan AM, et al. Fast assessment of stroke and transient ischaemic attack to prevent early recurrence (FASTER): a randomised controlled pilot trial. Lancet Neurol 2007;6:961-9.

14. Ernst E, Matrai A, Kollar L. Placebo-controlled, double-blind study of haemodilution in peripheral arterial disease. Lancet 1987;1:1449-51.

15. Papaioannou TG, Stefanadis C. Vascular wall shear stress: basic principles and methods. Hellenic J Cardiol 2005;46:9-15.

16. Jung LY, Lee SR, Jung JM, Kim YS, Lee SH, Rhee KS, et al. Rosuvastatin reduces blood viscosity in patients with acute coronary syndrome. Korean Circ J 2016;46:147-53.

17. Lee $\mathrm{CH}$, Jung $\mathrm{KH}$, Cho DJ, Jeong SK. Effect of warfarin versus aspirin on blood viscosity in cardioembolic stroke with atrial fibrillation: a prospective clinical trial. BMC Neurol 2019;
19:82.

18. Rosenson RS. Treatment with aspirin and dipyridamole is more effective than aspirin in reducing low shear blood viscosity. Microcirculation 2008;15:615-20.

19. Stäubli M, Reinhart W, Straub PW. Blood viscosity and red cell deformability after aspirin in vivo. A double-blind controlled trial. Atherosclerosis 1982;41:167-70.

20. Hitosugi M, Niwa M, Takatsu A. Changes in blood viscosity by heparin and argatroban. Thromb Res 2001;104:371-4.

21. Ciuffetti G, Lombardini R, Pirro M, Lupattelli G, Mannarino E. Clopidogrel: hemorheological effects in subjects with subclinical atherosclerosis. Clin Hemorheol Microcirc 2001;25:31-9.

22. Dawson DL, Zheng Q, Worthy SA, Charles B, Bradley DV Jr. Failure of pentoxifylline or cilostazol to improve blood and plasma viscosity, fibrinogen, and erythrocyte deformability in claudication. Angiology 2002;53:509-20.

23. Thomas DJ, Marshall J, Russell RW, Wetherley-Mein G, du Boulay GH, Pearson TC, et al. Effect of haematocrit on cerebral blood-flow in man. Lancet 1977;2:941-3. 